\title{
Evolving therapeutic concepts in prostate cancer based on genome-wide analyses (Review)
}

\author{
BJÖRN GEORGI $^{1 *}$, NINA KORZENIEWSKI ${ }^{2 *}$, BORIS HADASCHIK ${ }^{1}$, CARSTEN GRÜLLICH $^{3}$, WILFRIED ROTH ${ }^{4}$, \\ HOLGER SÜLTMANN $^{5}$, SASCHA PAHERNIK ${ }^{1}$, MARKUS HOHENFELLNER ${ }^{1}$ and STEFAN DUENSING $^{1,2}$ \\ ${ }^{1}$ Department of Urology, ${ }^{2}$ Section of Molecular Urooncology, Department of Urology, University of Heidelberg \\ School of Medicine; ${ }^{3}$ National Center for Tumor Diseases, Department of Medical Oncology; ${ }^{4}$ Department of Pathology, \\ University of Heidelberg School of Medicine; ${ }^{5}$ Division for Cancer Genome Research, National Center for Tumor Diseases \\ and German Cancer Research Center, D-69120 Heidelberg, Germany
}

Received June 3, 2014; Accepted July 9, 2014

DOI: 10.3892/ijo.2014.2567

\begin{abstract}
Treatment of castration resistant prostate cancer (CRPC) continues to represent a major urooncological challenge due to tumor heterogeneity and the inevitable development of therapy resistance. Although androgen deprivation therapy retains an important role in the management of CRPC, recent evidence suggests that a broader spectrum of therapeutic targets may improve patient response and delay development of advanced disease. Genome-wide analyses have identified four major signaling nodes that are most frequently altered in prostate cancer: i) the androgen receptor (AR); ii) the PI3K pathway; iii) the Ras/Raf/MEK/ERK pathway; and iv) the retinoblastoma protein (pRB) signaling pathway. Extensive crosstalk and redundancy exists between these signaling pathways, which underscores the need for combination therapies. There are several novel AR pathway inhibitors currently in clinical use. Clinical trials are being performed on single-agent PI3K inhibitors with some success in tumors with genetically altered PI3K components. MEK/ERK inhibitors are also in clinical trials and the importance of $\mathrm{pRB}$ inactivation in prostate cancer is becoming more widely recognized. A greater understanding of the effects of single agent therapy on compensatory signaling pathway activation that can potentially thwart antitumoral responses is urgently needed and will provide additional insight into the mechanism of therapy resistance and how to further delay the progression to lethal disease.
\end{abstract}

Correspondence to: Professor Stefan Duensing, Section of Molecular Urooncology, Department of Urology, University of Heidelberg School of Medicine, Medical Faculty Heidelberg, Im Neuenheimer Feld 517, D-69120 Heidelberg, Germany

E-mail: stefan.duensing@med.uni.heidelberg.de

${ }^{*}$ Contributed equally

Key words: prostate cancer, genome, signaling pathways, combination therapy

\section{Contents}

1. Introduction

2. Androgen receptor signaling

3. Pharmacological inhibition of androgen receptor signaling action

4. Phosphatidylinositol-3-kinase (PI3K) signaling

5. Pharmacological inhibition of PI3K signaling

6. Ras/Raf/MEK/ERK signaling

7. Pharmacological inhibition of the Ras/Raf/MEK/ERK pathway

8. Retinoblastoma protein (pRB) pathway

9. Pharmacological inhibition of $\mathrm{pRB}$ signaling

10. Conclusions and outlook

\section{Introduction}

Prostate cancer is the most common non-cutaneous malignancy in males causing one in three tumor-associated deaths in Germany and the second most common cause of cancer-related death in the United States (1) (Krebs in Deutschland; www.krebsdaten.de). Androgen deprivation therapy is the cornerstone of the urooncological management of advanced hormone-sensitive prostate cancer (2). However, patients inevitably enter a castration resistant stage in which tumor progression occurs despite androgen deprivation. These metastatic castration resistant prostate cancer (mCRPC) patients have, until recently, received chemotherapy (docetaxel, mitoxantrone) as first line therapy $(3,4)$. However, the introduction of novel and highly potent anti-androgens such as abiraterone or enzalutamide has led to a shift in the clinical practice and these agents are now also being used in chemo-naive mCRPC patients $(5,6)$. Nevertheless, treatment of mCRPC remains a major challenge due to the heterogeneity of the disease and the inevitable development of therapy resistance. It is therefore of importance to better understand the biology of prostate cancer progression in order to develop improved therapeutic strategies. 
Recent advances in comprehensive genomic profiling have identified four major signaling nodes that are most frequently altered in prostate cancer: i) the androgen receptor (AR) signaling pathway; ii) the PI3K pathway; iii) the Ras/Raf/MEK/ERK pathway; and iv) the retinoblastoma protein (pRB) signaling pathway (7). These pathways were altered at high frequencies in both primary tumors and metastatic samples in an exemplary study (7). The AR was identified as the most commonly mutated gene in prostate cancer with $56-100 \%$ of tumors examined containing mutated AR. Loss of PTEN, a negative regulator of the PI3K pathway, is a hallmark of prostate cancer and occurs in $42-100 \%$ of the tumors analyzed. The Ras/Raf/MEK/ERK pathway was altered in $43-90 \%$ of tumors and the pRB signaling pathway was altered in $34-74 \%$ of prostate cancers (7).

Importantly, extensive crosstalk and redundancy exists between these signaling pathways leading to the hypothesis that therapy resistance may readily develop when only one of the four pathways is targeted (7-9). This suggests that developing either novel therapeutic agents, or therapeutic regimes, that target a broader spectrum of pathway components may improve the clinical benefit of systemic therapy (Table I). In this review, we highlight the most commonly altered signaling pathways in advanced prostate cancer which need to be taken into account for the development of such new rational therapeutic strategies (Fig. 1).

\section{Androgen receptor signaling}

Androgen signaling is mediated through the androgen receptor (AR), a ligand-activated transcription factor that is the main therapeutic target of first-line therapies for advanced prostate cancer. The inactive form of the AR resides within the cytoplasm. Binding of androgens to the receptor leads to receptor activation and translocation into the nucleus, where the transcription of androgen-dependent genes is induced (10). Androgen-deprivation therapy (or chemical castration) is an effective first-line therapy for metastatic prostate cancer, but despite good initial responses, castrateresistant prostate cancer commonly develops leading to tumors that are insensitive to androgen ablation (11).

Castration resistance can develop through several mechanisms, most commonly by gene amplification, activating mutations of the AR, or expression of AR splice variants which promotes maintenance of AR activity and signaling capabilities at castrate hormone levels (7,12-15). Interestingly, AR gene amplification has been detected in $28 \%$ of CRPCs after androgen ablation, but has not been observed in tumor samples without prior exposure to androgen deprivation (12). Receptor hypersensitivity to androgens can also occur in CRPC through increased receptor stability, enhanced nuclear localization and overexpression of nuclear co-activators $(16,17)$. Furthermore, AR point mutations have been demonstrated to confer AR promiscuity leading to an activation by non-androgen ligands such as progesterone and estradiol $(18,19)$. These observations suggest a selective pressure to maintain AR-mediated signaling in CRPC despite androgen deprivation conditions.

Based on this fact, CRPC remains to a certain extent sensitive to second-generation AR antagonists and hormone
Table I. Inhibitors of the AR, PI3K, Ras/Raf/MEK/ERK and RB pathway.

\begin{tabular}{lc}
\hline Agent & Pathway inhibition \\
\hline Abiraterone & AR \\
Enzalutamide & AR \\
ARN 509 & AR \\
CFG920 & AR \\
Ortenerol (TAK700) & AR \\
BKM120 & PI3K \\
PX866 & PI3K \\
BEZ235 & PI3K/mTOR \\
GDC-0980 & PI3K/mTOR \\
MK2206 & AKT \\
GDC-0068 & AKT \\
Everolimus & TORC1 \\
Temsirolimus & TORC1 \\
PD98059 & MEK \\
U0126 & MEK \\
PD184352 (CI-1040) & MEK \\
PD0325901 & MEK \\
Selumetinib & MEK \\
RDEA119 & MEK \\
SL327 & MEK \\
Pimasertib (AS-703026) & MEK \\
BIX 02188 & MEK \\
BIX 02189 & MEK \\
AZD8330 & MEK \\
TAK-733 & MEK \\
Trametinib (GSK1120212) & MEK \\
AZD 5438 & CDKs \\
Seliciclib & CDKs \\
Flavopiridol & CDKs \\
PD0332991 & CDKs \\
LEE011 & CDK4/6 \\
\hline CDK4/6 \\
\hline (n)
\end{tabular}

Modified after $(37,77)$.

synthesis blockers, which includes drugs that target AR signaling at the androgen production and conversion level and at the receptor level such as abiraterone or enzalutamid (20). However, it has been shown that inhibition of the AR receptor can lead to activation of other signaling pathways via crosstalk, such as the PI3K pathway. For example, AR blockade can lead to a reduced expression of the AR-responsive immunophilin FBPK5, a chaperone for the AKT phosphatase PHLPP, and ultimately an increased level of phosphorylated AKT, a downstream target of PI3K (11). Further, non-ligand mediated activation of AR signaling has been shown to be induced through crosstalk with other oncogenic pathways such as the mitogen-activated protein kinase (MAPK) pathway $(21,22)$. 


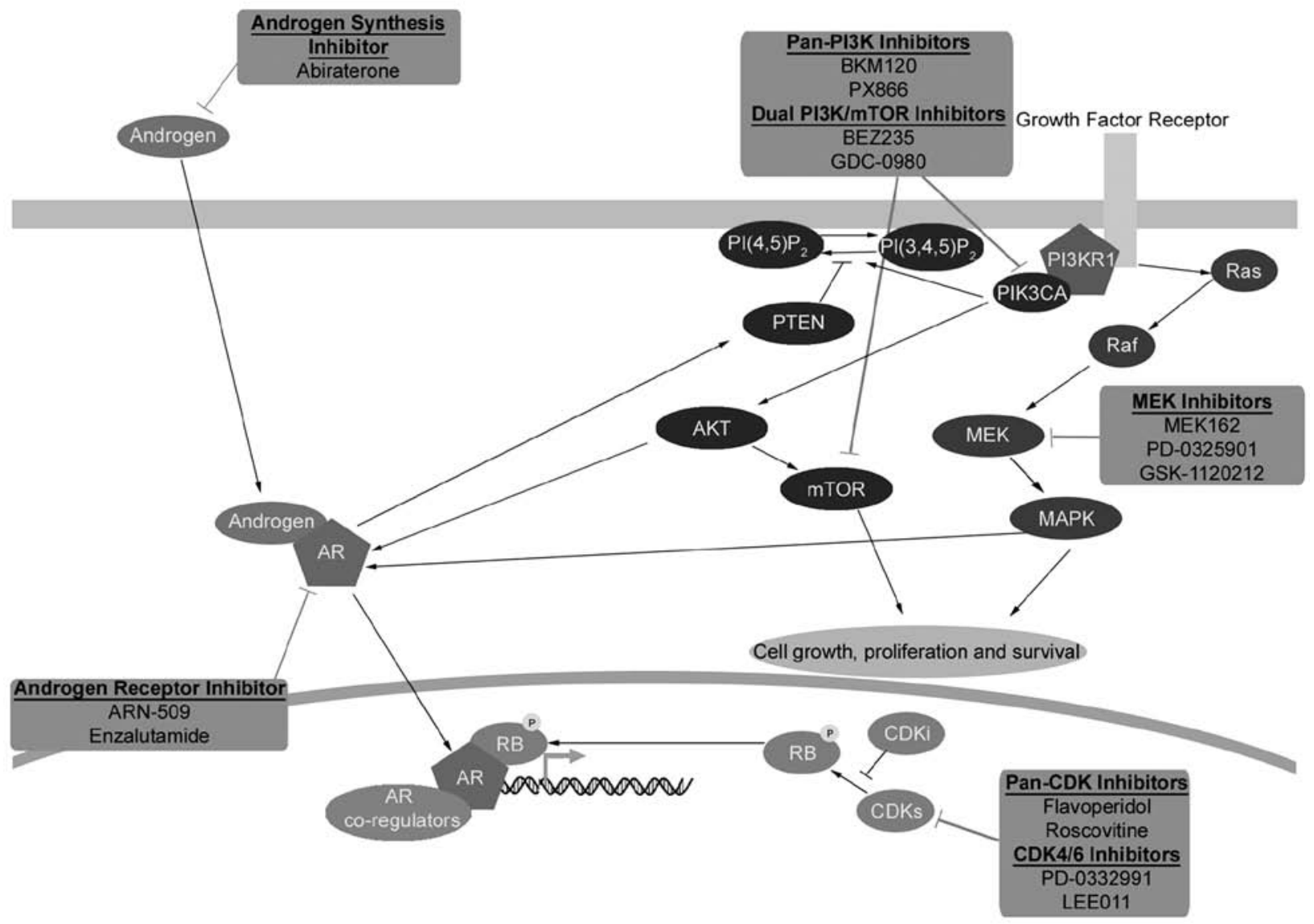

Figure 1. AR, PI3K, Ras/Raf/MEK/ERK and pRB pathway crosstalk and pharmacological inhibition. Recent advances in genomic profiling have identified four major signaling pathways that are most frequently altered in prostate cancer: i) the androgen receptor (AR) signaling pathway; ii) the PI3K pathway; iii) the Ras/Raf/MEK/ERK pathway; and iv) the retinoblastoma (pRB) signaling pathway. Extensive crosstalk and redundancy exists between these signaling pathways suggesting that devising a therapeutic regime that targets more than one of these pathways may provide a clinical advantage. Androgen signaling is mediated through AR, a ligand-activated transcription factor that is the main therapeutic target of first-line therapies for advanced prostate cancer. The inactive form of the AR resides within the cytoplasm, binding of androgens to the receptor leads to its nuclear translocation and transcription of androgen-dependent genes. Inhibition of the AR receptor can lead to activation of other signaling pathways such as the PI3K pathway through pathway crosstalk. The PI3K pathway is the second most frequently deregulated signaling pathway in prostate cancer behind only the AR signaling pathway. The PI3K pathway is normally activated following growth factor stimulation of receptor tyrosine kinases ultimately resulting in the activation of AKT, the central effector of many downstream signaling pathways regulating protein synthesis, cell cycle, cell death, cell growth and cell survival. AKT activation can be reversed by PTEN, which negatively regulates PI3K pathway activation. Another important target of the PI3K signaling pathway is the serine/threonine kinase mTOR (mammalian target of rapamycin). The Ras/Raf/MEK/ERK signaling pathway regulates fundamental cellular processes, including proliferation, differentiation and cell survival and is frequently activated in several cancer types, including prostate cancer. The PI3K and Ras/Raf/MEK/ERK pathways have been shown to interact extensively and frequent co-activation of these two pathways has been observed in prostate cancer. Ras/Raf/MEK/ERK signaling is stimulated by growth factor receptor activation of the small GTPase Ras, which in turn activates the protein kinase Raf leading to the activation of the dual specificity MAPK kinase (MEK1/MEK2), and finally phosphorylation of extracellular signal-regulated kinases (ERK1/ERK2). Ras/Raf/MEK/ERK pathway can sustain the transcription of androgen responsive genes, and therefore prostate cancer cell proliferation, even when the AR is inhibited. Retinoblastoma (RB1) is a tumor suppressor gene with somatic alterations in multiple cancers whose gene product (pRB) inhibits cell cycle progression. pRB activity is predominately controlled by cell cycle regulated cyclin-dependent kinase (CDK) activity. When CDK activity is inhibited, pRB remains in a hypophosphorylated and active state repressing E2F-regulated gene transcription and inhibiting cell cycle progression. Inactivation of pRB prevents cells from restraining cellular proliferation and leads to the aberrant expression of E2F-responsive genes. Dysregulation of E2F binding motif-containing genes is a hallmark of CRPC. Pharmacological inhibitors of the different signaling pathways are shown.

\section{Pharmacological inhibition of androgen receptor signaling action}

Abiraterone. Chemical castration leads to decreased production of testicular androgens but adrenal glands and even prostate cancer tissue can continue to produce androgens, which contributes to continued prostate cancer cell growth despite castrate level of androgens. Synthesized in the early 1990s, and approved for use in CRPC patients in 2011, abiraterone treatment promotes a global blockade of androgen production through irreversible inhibition of $17 \alpha$-hydroxylase/C17,20 lyase (CYP17), a key enzyme involved in androgen synthesis. CYP17 is expressed in testicular, adrenal, and prostatic tumor tissues and inhibition of CYP17 results in a profound decrease of circulating androgens. Currently, abiraterone is used in mCRPC patients both prior to and post chemotherapy with or without mild symptoms to delay symptomatic disease progression $(23,24)$. Side effects following abiraterone treatment include hypertension, decreased serum potassium, edema and increased adrenocorticotropic hormone (ACTH) release. Other frequent adverse events include fatigue, fluid retention, hypokalemia, hypertension, cardiac disorders and liver enzyme increases $(24,25)$. 
Combination therapy with other androgen signaling pathway inhibitors, albeit with different mechanisms of action, for example enzalutamide and/or other therapeutic agents, are currently ongoing or in the planning phase (26).

Enzalutamide. Enzalutamide affects multiple steps in the AR signaling pathway including: i) competitive inhibition of androgen binding to the AR; ii) inhibition of nuclear translocation of the AR into the nucleus; iii) reduction of AR association with DNA; and iv) in vitro suppression of growth and induction of apoptosis in cell lines with AR gene amplifications (27).

Enzalutamid is well tolerated, most frequent side-effects reported were fatigue $(33.6 \%)$, cardiac disorder $(6.1 \%)$, myocardial infarction (0.3\%), abnormalities like AST/ALT increase and bilirubin increase (1\%). Seizures were rare and occurred in $0.6 \%$ of patients (28).

Enzalutamide is used in the post-chemotherapy setting and was found to improve progression-free survival (PFS), quality of life (QOL) and overall survival (OS) (29). Results of the PREVAIL phase III trial showed a significantly improved PFS and OS also in chemotherapy-naive patients (6).

$A R N-509 . \mathrm{ARN}-509$ is a novel AR receptor antagonist that, unlike bicalutamide, does not show any agonist functions in the context of CRPC with AR overexpression. ARN-509 inhibits AR nuclear translocation and transcriptional activity and a phase I trial showed a favorable safety and side effect profile $(26,30,31)$.

\section{Phosphatidylinositol-3-kinase (PI3K) signaling}

One of the key pathways essential tocell proliferation, survival, and metabolism is the phosphatidylinositol 3-kinase (PI3K) pathway (32). The PI3K pathway is the second most frequently deregulated signaling pathway in prostate cancer, behind only the AR signaling pathway (7). The PI3K pathway is normally activated following growth factor stimulation of receptor tyrosine kinases ultimately resulting in the conversion of membrane phosphatidylinositol-bis-phosphate $(\mathrm{PI}(3,4) \mathrm{P} 2$; PIP2) to phosphatidylinositol-tri-phosphate $(\mathrm{PI}(3,4,5) \mathrm{P} 3$; PIP3) by PI3K. This conversion of PIP2 to PIP3 by PI3K can be reversed by the tumor suppressor phosphatase and tensin homolog deleted on chromosome ten (PTEN) phosphatase, which functions to negatively regulate PI3K pathway activation. The formation of PIP3 mediates the activation of AKT, which is the central effector of many downstream signaling pathways regulating protein synthesis, cell cycle, cell death, cell growth and cell survival (32).

Another important target of the PI3K signaling pathway is the serine/threonine kinase mTOR (mammalian target of rapamycin). mTOR occurs in two complexes, the TORC1 complex (mTOR bound to Raptor) and the mTORC2 complex (mTOR bound to Rictor). Both mTORC1 and mTORC2 are substrates of AKT: when activated, mTORC1 regulates protein translation, while mTORC2 can phosphorylate AKT and provide positive feedback to this branch of the signaling network (33).

Approximately $34-40 \%$ of primary and $74-100 \%$ of metastatic prostate cancers harbor alterations of components of the PI3K pathway, generally promoting aberrant pathway activation (7). These genetic aberrations frequently involve loss-of-function mutations, deletions, or epigenetic silencing of PTEN, a negative regulator of PI3K, and/or activating mutations in PIK3CA, the catalytic p110 $\alpha$ kinase subunit (7,34-36).

Importantly, it has recently been shown that inhibition of the PI3K pathway in PTEN-deficient prostate cancer activates AR signaling by relieving feedback inhibition of the receptor tyrosine kinases HER2 and HER3. Conversely, inhibition of AR signaling was shown to activate AKT signaling through a reduced expression of the AKT phosphatase PHLPP (11). Reciprocal feedback regulation of the PI3K and AR pathways provides a compelling explanation for the poor efficacy of single-pathway inhibition therapy, for instance inhibition of the AR pathway alone, in PTEN-null cancers and the substantially improved antitumoral efficacy of combined PI3K/AR pathway inhibition. This pathway crosstalk may also be partially responsible for induction of castration resistance, which further underscores the importance of developing PI3K pathway targeting agents for the treatment of prostate cancer patients.

\section{Pharmacological inhibition of PI3K signaling}

The PI3K pathway is target-rich and a number of efforts have been made to exploit this fact for anticancer therapy. The PI3Ks are grouped into three classes of enzymes (I-III), leading to the development of both pan- and isoform-specific $\mathrm{PI} 3 \mathrm{~K}$ inhibitors as well as dual PI3K/TORC1/2 inhibitors (37). Several substances are currently under investigation as single agents or in combination with abiraterone (7-9).

The pan-PI3K inhibitor BKM120 (buparlisib) is currently under investigation in men with CRPC as single agent and in combination with abiraterone in two clinical trials (NCT01385293, NCT01634061). Treatment-related adverse events in BKM120-treated patients were most commonly fatigue, nausea, rash, hyperglycemia, diarrhea, anorexia and mood alterations (37-39). The latter underlines the need for close observation for psychiatric symptoms in patients treated with PI3K inhibitors that are able to cross the blood-brain barrier. Metabolic adverse events were most often reversible and not acutely toxic (40).

Isoform-specific PI3K inhbitors may be particularly relevant in patients in which PIK3CA is mutated (up to 16\%) but redundancy between different isoform needs to be considered (7). Dual PI3K/mTOR inhibitor such as BEZ235 or GDC-0980 lead to a profound inhibition of PI3K signaling and and have so far been well-tolerated. BEZ235 or GDC-0980 in combination with abiraterone is currently under investigation in a phase I/II clinical trials in men with mCRPC (NCT01717898). AKT inhibitors such as perifosine have so far not led to significant clinical responses $(37,41)$. Again, combination therapies between antihormonal substances and PI3K inhibitors appear to be the most promising avenues for future drug development and results from ongoing phase II trials will be instrumental to corroborate this notion. Inhibitors of TORC1 such as rapamycin and its analogs everolimus or temsirolimus did not show significant antitumoral effects when used as single agents in men with CRPC $(37,41)$.

\section{Ras/Raf/MEK/ERK signaling}

The Ras/Raf/MEK/ERK signaling pathway regulates fundamental cellular processes, including proliferation, differ- 
entiation, and cell survival and is frequently activated in several cancer types including prostate cancer. Increased activation of this pathway correlates with a poor prognosis and tumor invasiveness (42-44). Ras/Raf/MEK/ERK signaling is stimulated by growth factor receptor activation of the small GTPase Ras, which in turn activates the protein kinase Raf leading to the activation of the dual specificity MAPK kinase (MEK1/MEK2), and finally phosphorylation of extracellular signal-regulated kinases (ERK1/ERK2) (45). The PI3K and Ras/Raf/MEK/ ERK pathways have been shown to interact extensively and frequent co-activation of these two pathways has been observed in prostate cancer (46). Both pathways share common activation signals, such as receptor tyrosine kinase mediated activation, and also appear to provide compensatory signaling when one or the other is inhibited (47). Approximately $43 \%$ of primary and $90 \%$ of metastatic prostate tumors were found to harbor genetic alterations of the Ras/Raf/MEK/ERK pathway (7).

Although members of the Ras family are rarely mutated in general as well as in prostate cancer, the expression of important growth factor receptors promoting Ras activation, such as EGFR, FGFR and PDGFR, is frequently upregulated in prostate cancers (48). Further, ERK1/ERK2 activation is associated with increasing Gleason score and tumor stage (49). This is consistent with a functional role for the Ras/Raf/MEK/ERK signaling pathway as prostate cancer progresses to a more advanced, androgen-independent stage.

An activated Ras/Raf/MEK/ERK pathway could therefore provide a selective advantage to tumor cells under androgendeprivation pressure This notion is corroborated by the finding that the Ras/Raf/MEK/ERK pathway can sustain the transcription of androgen-responsive genes, and therefore prostate cancer cell proliferation, even when the AR is inhibited $(49,50)$.

\section{Pharmacological inhibition of the Ras/Raf/MEK/ERK pathway}

Efforts to target Ras directly have not been successful in the clinic to date, but recent clinical trials with Raf and MEK inhibitors have suggested that targeting these downstream Ras effectors could be promising.

Single agent therapy with MEK inhibitor alone showed a compensatory upregulation of the PI3K pathway as well as several others including NF- $\kappa \mathrm{B}$ and hedgehog as an expression of cellular pro-survival mechanisms (51). Dual inhibition of both MEK and PI3K/mTOR signaling increases apoptosis in cell lines and dual inhibition of AKT/mTOR and ERK has been demonstrated to lead to effective growth inhibition in mouse models of prostate cancer $(46,52)$. Furthermore, ERK inhibition was found to enhance docetaxel-induced cytotoxicity in androgen-independent prostate cancer cells (63).

Phase III trials have been performed with GSK-1120212 (trametinib), which is an FDA-approved MEK1/2 inhibitor, for metastatic melanoma showing a favorable safety profile. Main adverse events were acneiform skin alterations, diarrhea, peripheral edema, hypertension and transient mild cardiac dysfunction. Most toxicities did not require drug discontinuation (54-57). Trials are going to be initiated in patients with different tumor entities (58). Other MEK inhibitors such as and MEK162 (binimetinib) and PD-0325901 are likewise in phase I/II clinical trials.

\section{Retinoblastoma protein (pRB) pathway}

The retinoblastoma gene (RB1) is a tumor suppressor gene with somatic alterations in multiple cancers whose protein product (pRB) inhibits cell cycle progression (59). In prostate cancer, $\mathrm{pRB}$ inactivation through genomic deletion of RB1 has been reported in approximately $20-60 \%$ of tumors and is associated with transition to CRPC and poor clinical outcome $(60,61)$. pRB activity is predominately controlled by cell cycle regulated cyclin-dependent kinase (CDK) activity (58). When CDK activity is inhibited, pRB remains in a hypophosphorylated and active state repressing E2F-mediated gene transcription thereby inhibiting cell cycle progression (58). Inactivation of $\mathrm{pRB}$ prevents cells from restraining cellular proliferation and leads to an aberrant expression of E2F-responsive genes (62). Overexpression of cyclins also contributes to the deregulation of $\mathrm{pRB}$ function in prostate cancer $(7,63,64)$. Remarkably, CRPC tissue has been found to show an altered repertoire of AR binding sites that were enriched for E2F motifs, which can lead to cyclin/CDK hyperactivity, pRB inactivation and uncontrolled proliferation (61).

Although it was demonstrated that pRB-deficient tumors respond poorly to hormone therapy, increasing evidence suggests that tumors with reduced $\mathrm{pRB}$ expression exhibit a more beneficial initial response to chemotherapy (65). Thus, RB1/pRB status could be a predictive marker of response to chemotherapy, with a potential to influence clinical decision-making, and a potential biomarker of transition to castration resistance. In addition, treatment with anti-androgens can lead to more aggressive tumors with neuroendocrine differentiation which is often associated with a loss of $\mathrm{pRB}$ protein expression (66).

\section{Pharmacological inhibition of pRB signaling}

Numerous studies have explored CDK inhibitors to target tumor cells with inactivated pRB and E2F-induced cyclin/CDK hyperactivity. The pan-CDK (CDK1, CDK2, CDK4/6) inhibitor flavoperidol was found to enhance apoptosis in androgenindependent PC-3 prostate cancer cells (67). An increase in apoptosis and a decrease in angiogenesis was detected when combined with docetaxel in a mouse model of prostate cancer $(68,69)$. In addition, it has been shown that CDK1 can phosphorylate the AR at serine 5115 which was associated with unfavorable patient outcome. The CDK inhibitor roscovitine was found to reduce AR phosphorylation, which could be exploited clinically (70). A selective CDK4/6 inhibitor, PD-0332991 (palbociclib), showed a growth suppressive effect in prostate cancer xenografts. Phase I studies in colorectal cancer and multiple myeloma are ongoing (71). The CDK4/6 inhibitor LEE011 is currently under investigation in several phase I and II trials for advanced solid tumor, breast cancer, lymphoma and melanoma (72). Possible side effects known from clinical trials with roscovitine are nausea, vomiting, transient elevations in serum creatinine and liver enzymes.

\section{Conclusions and outlook}

Due to extensive crosstalk between signaling pathways such as the AR and PI3K pathways, PI3K and Ras/Raf/MEK/ERK pathway and $\mathrm{pRB}$ and AR signaling, single-agent therapy is 
likely to result in an activation of mechanisms that thwart the antitumoral response, lead to drug resistance and ultimately treatment failure. Future drug and clinical trial development therefore needs to reflect the extensive feedback mechanisms that exist between the four most frequently altered signaling nodes in prostate cancer. However, there are a number of questions and concerns that need to be addressed. First and foremost, it will be important to determine the safety, tolerability and the actual clinical effectiveness of treatment regimen that encompass two or more pathway-targeting drugs. Given that unexpected and paradoxical survival pathway activation in response to targeted agents has been reported in other malignancies (73-76), it is possible that, depending on the genetic background and intratumoral heterogeneity, combination therapies that have been found to be effective in vitro and in preclinical models may not show the same efficacy when used in cancer patients.

Second, toxicity profiles for combination targeted therapies need to be carefully analyzed and weighed against the clinical benefit. Whether and to what extent a dose reduction of individual compounds is permissable without impeding the oncological effectiveness as it has been shown for 'classical' chemotherapeutic agents remains to be determined. Nonetheless, it is worth remembering that combination drug therapies were a major breakthrough in the chemotherapeutic treatment of several malignancies including Hodgkin's lymphoma and several others. So far, toxicity profiles of newer generation pathway inhibitors appear to be manageable but unexpected pharmacological interactions and resistance mechanisms always need to be considered.

Third, regulatory limitations are a major concern. Effective drugs may not be manufactured by the same pharmaceutical company and may lack approval in certain geographic regions. How to deal with this problem and whether multipathway targeting drugs are an attainable solution remains to be determined.

Nonetheless, from a purely biological point of view, novel treatment strategies for prostate cancer need to reflect the highly disorganized and profoundly interconnected signaling landscape that has emerged from genome-wide analyses.

\section{Acknowledgements}

Work in the authors' laboratory is supported by the Medical Faculty Heidelberg.

\section{References}

1. Siegel R, Naishadham D and Jemal A: Cancer statistics, 2013 CA Cancer J Clin 63: 11-30, 2013.

2. Prostate Cancer Trialists' Collaborative Group: Maximum androgen blockade in advanced prostate cancer: an overview of the randomised trials. Lancet 355: 1491-1498, 2000.

3. Berthold DR, Pond GR, Soban F, de Wit R, Eisenberger M and Tannock IF: Docetaxel plus prednisone or mitoxantrone plus prednisone for advanced prostate cancer: updated survival in the TAX 327 study. J Clin Oncol 26: 242-245, 2008.

4. Tannock IF, de Wit R, Berry WR, et al: Docetaxel plus prednisone or mitoxantrone plus prednisone for advanced prostate cancer. N Engl J Med 351: 1502-1512, 2004.

5. Ryan CJ, Smith MR, de Bono JS, et al: Abiraterone in metastatic prostate cancer without previous chemotherapy. N Engl J Med 368: 138-148, 2013.
6. Beer TM, Armstrong AJ, Sternberg CN, et al: Enzalutamide in men with chemotherapy-naive metastatic prostate cancer (mCRPC): Results of phase III PREVAIL study. J Clin Oncol 32 (Suppl 4): abs. LBA1^, 2014.

7. Taylor BS, Schultz N, Hieronymus $\mathrm{H}$, et al: Integrative genomic profiling of human prostate cancer. Cancer Cell 18: 11-22, 2010.

8. Mulholland DJ, Kobayashi N, Ruscetti M, et al: Pten loss and RAS/MAPK activation cooperate to promote EMT and metastasis initiated from prostate cancer stem/progenitor cells. Cancer Res 72: 1878-1889, 2012.

9. Carver BS, Chapinski C, Wongvipat J, et al: Reciprocal feedback regulation of PI $3 \mathrm{~K}$ and androgen receptor signaling in PTEN-deficient prostate cancer. Cancer Cell 19: 575-586, 2011.

10. Bennett NC, Gardiner RA, Hooper JD, Johnson DW and Gobe GC: Molecular cell biology of androgen receptor signalling. Int J Biochem Cell Biol 42: 813-827, 2010.

11. Ryan CJ and Tindall DJ: Androgen receptor rediscovered: the new biology and targeting the androgen receptor therapeutically. J Clin Oncol 29: 3651-3658, 2011.

12. Koivisto P, Kononen J, Palmberg C, et al: Androgen receptor gene amplification: a possible molecular mechanism for androgen deprivation therapy failure in prostate cancer. Cancer Res 57: 314-319, 1997.

13. Edwards J, Krishna NS, Grigor KM and Bartlett JM: Androgen receptor gene amplification and protein expression in hormone refractory prostate cancer. Br J Cancer 89: 552-556, 2003.

14. Dehm SM, Schmidt LJ, Heemers HV, Vessella RL and Tindall DJ: Splicing of a novel androgen receptor exon generates a constitutively active androgen receptor that mediates prostate cancer therapy resistance. Cancer Res 68: 5469-5477, 2008.

15. Sun S, Sprenger CC, Vessella RL, et al: Castration resistance in human prostate cancer is conferred by a frequently occurring androgen receptor splice variant. J Clin Invest 120: 2715-2730, 2010.

16. Chen S, Xu Y, Yuan X, Bubley GJ and Balk SP: Androgen receptor phosphorylation and stabilization in prostate cancer by cyclin-dependent kinase 1. Proc Natl Acad Sci USA 103: 15969-15974, 2006.

17. Fujimoto N, Mizokami A, Harada S and Matsumoto T: Different expression of androgen receptor coactivators in human prostate. Urology 58: 289-294, 2001.

18. Taplin ME, Rajeshkumar B, Halabi S, et al: Androgen receptor mutations in androgen-independent prostate cancer: Cancer and Leukemia Group B Study 9663. J Clin Oncol 21: 2673-2678, 2003.

19. Sack JS, Kish KF, Wang C, et al: Crystallographic structures of the ligand-binding domains of the androgen receptor and its T877A mutant complexed with the natural agonist dihydrotestosterone. Proc Natl Acad Sci USA 98: 4904-4909, 2001.

20. Stein MN, Patel N, Bershadskiy A, Sokoloff A and Singer EA: Androgen synthesis inhibitors in the treatment of castration-resistant prostate cancer. Asian J Androl 16: 387-400, 2014.

21. Abreu-Martin MT, Chari A, Palladino AA, Craft NA and Sawyers CL: Mitogen-activated protein kinase kinase kinase 1 activates androgen receptor-dependent transcription and apoptosis in prostate cancer. Mol Cell Biol 19: 5143-5154, 1999.

22. Bakin RE, Gioeli D, Sikes RA, Bissonette EA and Weber MJ: Constitutive activation of the Ras/mitogen-activated protein kinase signaling pathway promotes androgen hypersensitivity in LNCaP prostate cancer cells. Cancer Res 63: 1981-1989, 2003.

23. Araujo JC, Trudel GC, Saad F, et al: Docetaxel and dasatinib or placebo in men with metastatic castration-resistant prostate cancer (READY): a randomised, double-blind phase 3 trial. Lancet Oncol 14: 1307-1316, 2013.

24. Cea R: Interim analysis results of COU-AA-302, a randomized, phase 3 study of abiraterone acetate (AA) in chemotherapienaive patients with metastatic castration-resistant prostate cancer (mCRPC). J Clin Oncol (Suppl 30): abs LBA4518, 2012.

25. De Bono JS, Logothetis CJ, Molina A, et al: Abiraterone and increased survival in metastatic prostate cancer. $N$ Engl J Med 364: 1995-2005, 2011

26. Sridhar SS, Freedland SJ, Gleave ME, et al: Castration-resistant prostate cancer: from new pathophysiology to new treatment. Eur Urol 65: 289-299, 2014.

27. Tran C, Ouk S, Clegg NJ, et al: Development of a secondgeneration antiandrogen for treatment of advanced prostate cancer. Science 324: 787-790, 2009. 
28. De Bono JS: Primary, secondary, and quality-of-life endpoint results from the phase III AFFIRM study of MDV3100, an androgen receptor signaling inhibitor J Clin Oncol (Suppl 30): abs 4519, 2012

29. Scher HI, Fizazi K, Saad F, et al: Increased survival with enzalutamide in prostate cancer after chemotherapy. N Engl J Med 367: 1187-1197, 2012

30. Clegg NJ, Wongvipat J, Joseph JD, et al: ARN-509: a novel antiandrogen for prostate cancer treatment. Cancer Res 72 : 1494-1503, 2012.

31. Rathkopf DE, Morris MJ, Fox JJ, et al: Phase I study of ARN-509, a novel antiandrogen, in the treatment of castrationresistant prostate cancer. J Clin Oncol 31: 3525-3530.

32. Wong KK, Engelman JA and Cantley LC: Targeting the PI3K signaling pathway in cancer. Curr Opin Genet Dev 20: 87-90, 2010.

33. LoPiccolo J, Blumenthal GM, Bernstein WB and Dennis PA: Targeting the PI3K/Akt/mTOR pathway: effective combinations and clinical considerations. Drug Resist Updat 11: 32-50, 2008.

34. Vlietstra RJ, van Alewijk DC, Hermans KG, van Steenbrugge GJ and Trapman J: Frequent inactivation of PTEN in prostate cancer cell lines and xenografts. Cancer Res 58: 2720-2723, 1998.

35. Verhagen PC, van Duijn PW, Hermans KG, et al: The PTEN gene in locally progressive prostate cancer is preferentially inactivated by bi-allelic gene deletion. J Pathol 208: 699-707, 2006.

36. Konishi N, Nakamura M, Kishi M, Nishimine M, Ishida E and Shimada K: Heterogeneous methylation and deletion patterns of the INK4a/ARF locus within prostate carcinomas. Am J Pathol 160: 1207-1214, 2002.

37. Bitting RL and Armstrong AJ: Targeting the PI3K/Akt/mTOR pathway in castration-resistant prostate cancer. Endocr Relat Cancer 20: R83-R99, 2013.

38. Ando Y, Inada-Inoue M, Mitsuma A, et al: Phase I dose-escalation study of buparlisib (BKM120), an oral pan-class I PI3K inhibitor, in Japanese patients with advanced solid tumors. Cancer Sci 105: 347-353.

39. Bendell JC, Rodon J, Burris HA, et al: Phase I, dose-escalation study of BKM120, an oral pan-class I PI3K inhibitor, in patients with advanced solid tumors. J Clin Oncol 30: 282-290, 2012.

40. Busaidy NL, Farooki A, Dowlati A, et al: Management of metabolic effects associated with anticancer agents targeting the PI3K-Akt-mTOR pathway. J Clin Oncol 30: 2919-2928, 2012.

41. Armstrong GT, Kawashima T, Leisenring W, et al: Aging and risk of severe, disabling, life-threatening, and fatal events in the childhood cancer survivor study. J Clin Oncol 32: 1218-1227, 2014.

42. McCubrey JA, Steelman LS, Chappell WH, et al: Roles of the Raf/MEK/ERK pathway in cell growth, malignant transformation and drug resistance. Biochim Biophys Acta 1773: 1263-1284, 2007.

43. McCubrey JA, Steelman LS, Abrams SL, et al: Roles of the Raf/MEK/ERK and PI3K/PTEN/AKT pathways in malignant transformation and drug resistance. Adv Enzyme Regul 46: 249-279, 2006

44. Ramsay AK, McCracken SR, Soofi M, et al: ERK5 signalling in prostate cancer promotes an invasive phenotype. Br J Cance 104: 664-672, 2011.

45. Kim EK and Choi EJ: Pathological roles of MAPK signaling pathways in human diseases. Biochim Biophys Acta 1802: 396-405, 2010

46. Kinkade CW, Castillo-Martin M, Puzio-Kuter A, et al: Targeting AKT/mTOR and ERK MAPK signaling inhibits hormone-refractory prostate cancer in a preclinical mouse model. J Clin Invest 118: 3051-3064, 2008

47. Shimizu T, Tolcher AW, Papadopoulos KP, et al: The clinical effect of the dual-targeting strategy involving PI3K/AKT/mTOR and RAS/MEK/ERK pathways in patients with advanced cancer. Clin Cancer Res 18: 2316-2325, 2012.

48. Drake JM, Graham NA, Stoyanova T, et al: Oncogene-specific activation of tyrosine kinase networks during prostate cancer progression. Proc Natl Acad Sci USA 109: 1643-1648, 2012.

49. Carey AM, Pramanik R, Nicholson LJ, et al: Ras-MEK-ERK signaling cascade regulates androgen receptor element-inducible gene transcription and DNA synthesis in prostate cancer cells. Int J Cancer 121: 520-527, 2007.

50. Weber MJ and Gioeli D: Ras signaling in prostate cancer progression. J Cell Biochem 91: 13-25, 2004.
51. Gioeli D, Wunderlich W, Sebolt-Leopold J, et al: Compensatory pathways induced by MEK inhibition are effective drug targets for combination therapy against castration-resistant prostate cancer. Mol Cancer Ther 10: 1581-1590, 2011

52. Steelman LS, Abrams SL, Shelton JG, et al: Dominant roles of the Raf/MEK/ERK pathway in cell cycle progression, prevention of apoptosis and sensitivity to chemotherapeutic drugs. Cell Cycle 9: 1629-1638, 2010.

53. Zelivianski S, Spellman M, Kellerman M, et al: ERK inhibitor PD98059 enhances docetaxel-induced apoptosis of androgen-independent human prostate cancer cells. Int J Cancer 107: 478-485, 2003.

54. Menzies AM and Long GV: Dabrafenib and Trametinib, alone and in combination for BRAF-mutant metastatic melanoma. Clin Cancer Res 20: 2035-2043, 2014

55. Schadendorf D, Amonkar MM, Milhem M, et al: Functional and symptom impact of trametinib versus chemotherapy in BRAF V600E advanced or metastatic melanoma: quality-oflife analyses of the METRIC study. Ann Oncol 25: 700-706

56. Anforth R, Liu M, Nguyen B, et al: Acneiform eruptions: A common cutaneous toxicity of the MEK inhibitor trametinib. Australas J Dermatol doi: 10.1111/ajd.12124, 2013 [Epub ahead of print].

57. Kim KB, Kefford R, Pavlick AC, et al: Phase II study of the MEK1/MEK2 inhibitor Trametinib in patients with metastatic BRAF-mutant cutaneous melanoma previously treated with or without a BRAF inhibitor. J Clin Oncol 31: 482-489, 2013.

58. Giacinti $\mathrm{C}$ and Giordano A: RB and cell cycle progression. Oncogene 25: 5220-5227, 2006

59. Sellers WR and Kaelin WG Jr: Role of the retinoblastoma protein in the pathogenesis of human cancer. J Clin Oncol 15: 3301-3312, 1997.

60. Grasso CS, Wu YM, Robinson DR, et al: The mutational landscape of lethal castration-resistant prostate cancer. Nature 487: 239-243, 2012.

61. Sharma A, Yeow WS, Ertel A, et al: The retinoblastoma tumor suppressor controls androgen signaling and human prostate cancer progression. J Clin Invest 120: 4478-4492, 2010.

62. Dyson N: The regulation of E2F by pRB-family proteins. Genes Dev 12: 2245-2262, 1998

63. Mashal RD,Lester S, Corless C, et al: Expression of cell cycle-regulated proteins in prostate cancer. Cancer Res 56: 4159-4163, 1996.

64. Drobnjak M, Osman I, Scher HI, Fazzari M and Cordon-Cardo C: Overexpression of cyclin D1 is associated with metastatic prostate cancer to bone. Clin Cancer Res 6: 1891-1895, 2000.

65. Aparicio A, Den RB and Knudsen KE: Time to stratify? The retinoblastoma protein in castrate-resistant prostate cancer. Nat Rev Urol 8: 562-568, 2011.

66. Tan HL, Sood A, Rahimi HA, et al: Rb loss is characteristic of prostatic small cell neuroendocrine carcinoma. Clin Cancer Res 20: 890-903, 2014.

67. Nathwani SM, Cloonan SM, Stronach M, et al: Novel microtubule-targeting agents, pyrrolo-1,5-benzoxazepines, induce cell cycle arrest and apoptosis in prostate cancer cells. Oncol Rep 24: 1499-1507, 2010.

68. Reiner T, de las Pozas A and Perez-Stable C: Sequential combinations of flavopiridol and docetaxel inhibit prostate tumors, induce apoptosis, and decrease angiogenesis in the Ggamma/ T-15 transgenic mouse model of prostate cancer. Prostate 66: $1487-1497,2006$

69. Gomez LA, de Las Pozas A and Perez-Stable C: Sequential combination of flavopiridol and docetaxel reduces the levels of X-linked inhibitor of apoptosis and AKT proteins and stimulates apoptosis in human LNCaP prostate cancer cells. Mol Cancer Ther 5: 1216-1226, 2006.

70. Willder JM, Heng SJ, McCall P, Adams CE, Tannahill C, Fyffe G, Seywright M, Horgan PG, Leung HY, Underwood MA and Edwards J: Androgen receptor phosphorylation at serine 515 by Cdk1 predicts biochemical relapse in prostate cancer patients. Br J Cancer 15: 139-148, 2013.

71. Gilbert MR, Dignam J, Pugh S, et al: Reply to m.C. Chamberlain. J Clin Oncol 32: 1634-1635, 2014.

72. Vici P, Capomolla E, Foggi P, et al: High activity of salvage treatment with biweekly paclitaxel-gemcitabine combination in heavily pretreated breast cancer patients. J Exp Clin Cancer Res 25: 39-44, 2006.

73. Wang X, Hawk N, Yue P, et al: Overcoming mTOR inhibitioninduced paradoxical activation of survival signaling pathways enhances mTOR inhibitors' anticancer efficacy. Cancer Biol Ther 7: 1952-1958, 2008 
74. Chu S, Holtz M, Gupta M and Bhatia R: BCR/ABL kinase inhibition by imatinib mesylate enhances MAP kinase activity in chronic myelogenous leukemia $\mathrm{CD} 4^{+}$cells. Blood 103: 3167-3174, 2004

75. Yu C, Krystal G, Varticovksi L, et al: Pharmacologic mitogenactivated protein/extracellular signal-regulated kinase kinase/ mitogen-activated protein kinase inhibitors interact synergistically with STI571 to induce apoptosis in Bcr/Abl-expressing human leukemia cells. Cancer Res 62: 188-199, 2002.
76. Packer LM, Rana S, Hayward R, et al: Nilotinib and MEK inhibitors induce synthetic lethality through paradoxical activation of RAF in drug-resistant chronic myeloid leukemia. Cancer Cell 20: 715-727, 2011.

77. Dickson MA and Schwartz GK: Development of cell-cycle inhibitors for cancer therapy. Curr Oncol 16: 36-43, 2009. 